\title{
PLANEAMIENTO ESTRATÉGICO EN LA DINÁMICA HUMANA Y ORGANIZACIONAL COMO PRODUCTO DE LOS PROCESOS COGNOSCITIVOS
}

\author{
José Luis Perea Rivera ${ }^{1}$ \\ Universidad Nacional Mayor de San Marcos, Perú \\ (RECIBIDO EL 10/01/2008, ACEPTADO EL 09/05/2008)
}

\begin{abstract}
RESUMEN
La temática del Planeamiento Estratégico con el advenimiento de la administración estratégica aparece como modelo de ajuste para la formulación e implementación de una estrategia que ayude a las organizaciones en establecer una ventaja competitiva, despertando gran interés para su aplicación en el ámbito organizacional en los campos de la administración y la ingeniería, es por ello que el propósito está orientado a plantear su aplicabilidad en el ámbito individual sea en los contextos de la dinámica personal y laboral de los profesionales de la salud, en razón que se observa y evidencia que la técnica en su operatividad depende del componente psicológico que está directamente relacionada con los procesos cognoscitivos del ser humano, principalmente el pensamiento. También plantea propuestas para su manejo y del modelo que asegure su sostenibilidad estructural modular ante la aparición de nuevas técnicas y modas administrativas; de igual manera, el utilizar el método de casos como la técnica que posibilita el entrenamiento del cerebro a pensar inteligentemente, y la herramienta útil para la toma de decisiones.
\end{abstract}

Palabras clave: Planeamiento estratégico, Procesos cognoscitivos, Pensamiento.

\begin{abstract}
The thematic one must like intention to approach the main processes of the management of the human resources in the organizations of production of goods and services (company, industry, institution) is of the public or deprived sector, that in its operational dynamics is present the psychological component. Also it treats his interrelation that under a systemic approach and in the global perspective towards the modernity of Century XXI within the agreement and the organizational change, serves as support in the systematization of the management; which it causes the consistency between the planning, the evaluation for aims of the continuous improvement, accreditation or certification of quality of his processes, and the pertinence with the demands of the social reality, economic and also political of the country.
\end{abstract}

Keywords: Organizacional change, Gathering, Habilitation, Qualification, Potentiality.

1 Docente auxiliar de la Facultad de Psicología de la Universidad Nacional Mayor de San Marcos. E-mail: pepeperea25@gmail.com 


\section{INTRODUCCIÓN}

La temática del Planeamiento Estratégico por su práctica está generalmente identificada en el campo de la administración, siendo de uso obligado en las organizaciones tanto en los países desarrollados como en vías de desarrollo, como una de las herramientas valiosas de gestión en la dinámica de las organizaciones.

De igual manera, se considera al Planeamiento Estratégico de gran utilidad en los diversos campos profesionales de la ingeniería y administración, resultando más que paradójico que en el trabajo de los profesionales de la salud, particularmente en el campo de la Psicología, no se le otorgue importancia, lo que a su vez restringe el alcance de su aplicabilidad en el plano existencial de la dinámica humana.

Esta realidad implica brindar un mayor conocimiento de esta herramienta de gestión para que su uso se proyecte al plano individual; por un lado, como un medio eficaz que les permita lograr los objetivos de su plan de vida mediante una dinámica humana eficiente y efectiva (misión), y del otro, para lograr con satisfacción el deseado estado ideal de éxito personal (visión).

Tratar esta temática en una perspectiva psicológica tiene como propósito facilitar la reflexión sobre el Planeamiento Estratégico, que para su elaboración y efectiva aplicación necesita como recursos-insumos a los trascendentales procesos cognoscitivos del pensamiento, percepción, memoria e imaginación.

\section{PRESENTACIÓN}

El contenido del trabajo tiene un marco referencial interdisciplinario, que exige en su desarrollo una explicitación focalizada sobre los procesos del planeamiento y el pensamiento en los contextos de la Administración y la Psicología, cuyo propósito es efectuar distinciones funcionales e interrelación interdisciplinario en su aplicación.

Este panorama posibilita delinear en un primer nivel de trabajo la naturaleza funcional del proceso cognoscitivo del pensamiento como recurso para la función administrativa, y en el segundo nivel, la naturaleza estructural del planeamiento como proceso en una perspectiva psicológica de los procesos cognoscitivos.

\section{PLANTEAMIENTO DE LA SITUACIÓN O PROBLEMA}

Partiendo del análisis de la dinámica organizacional, la temática del Planeamiento Estratégico como producto de los procesos cognoscitivos plantea su factibilidad de proyectarla hacia la dinámica humana, a fin de que su uso en el plano individual sirva como un medio eficaz para lograr los objetivos del plan de vida personal en forma eficiente y efectiva (misión), y que ese logro le permita disfrutar con satisfacción el deseado estado ideal del éxito personal (visión). 


\section{MARCO TEÓRICO REFERENCIAL}

Para el primer nivel de trabajo que aborda la naturaleza funcional del proceso cognoscitivo del pensamiento como recurso para la función administrativa de la planeación, tomaremos del contexto de la Administración como referentes de entrada lo que trata la Teoría de las Organizaciones (TO) y la Teoría General de la Administración-TGA (1) con respecto a la dimensión del pensamiento.

La Teoría de las Organizaciones trata del "estudio de las organizaciones en general", y la Teoría General de la Administración trata el "estudio de la administración en general, independientemente de si ésta se aplica en organizaciones con ánimo de lucro (empresas) o en aquellas que no lo tienen. La TGA estudia la administración de las organizaciones".

El enfoque de la Teoría General de la Administración tuvo una gradual y creciente ampliación, desde el clásico -pasando por el humanístico, neoclásico, estructuralista y behaviorista- hasta el enfoque sistémico.

El enfoque clásico tomó los tres principios intelectuales predominantes en todas las ciencias desde comienzo del siglo XX: el reduccionismo, el mecanicismo y el pensamiento analítico.

Estos principios fueron sustituidos con la Teoría General de Sistemas -TGS- Cit. (a), por los principios del expansionismo, la teleología y el pensamiento sintético.

En el pensamiento sintético "el fenómeno se explica por el rol que desempeña en dicho sistema, en este sentido el enfoque sistémico está más interesado en unir las cosas que separarlas".

Los tres principios de la TGS propiciaron el surgimiento de la cibernética. Cit. (b)

Para complementar este primer nivel de trabajo, se ha condensado con criterio holístico los planteamientos de las fuentes básicas en el contexto de la Psicología que nos permita exponerlos a fin de lograr un mejor entendimiento y comprensión en la relación de la naturaleza funcional del pensamiento como recurso-insumo para la función administrativa de la planeación.

En este sentido se ha tomado de sendas fuentes la información que sirve de marco referencial, así tenemos (2), (3) y (4):

- El pensamiento es el proceso psíquico socialmente condicionado de búsquedas y descubrimiento de lo nuevo y está indisolublemente ligado al lenguaje.

- El pensamiento está orientado hacia un objetivo. La necesidad de pensamiento surge ante todo, cuando en el proceso de la vida y la práctica de la persona aparece un nuevo fin, nuevas situaciones y condiciones de la actividad, un problema.

- Esta actividad cognoscitiva parte de las sensaciones y percepciones, luego puede suceder el tránsito al pensamiento; cualquier pensamiento, incluso el más desarrollado, mantiene siempre conexión con el conocimiento sensorial (sensaciones, percepciones y representaciones). 
- Esta actividad recibe todo el "material" solo desde una fuente: el conocimiento sensorial; de esta manera el pensamiento empieza donde el conocimiento sensorial se hace insuficiente e incluso impotente; es decir, el pensamiento continúa y desarrolla el "trabajo cognoscitivo" de las sensaciones, percepciones y representaciones, superando ampliamente sus límites.

* El pensamiento humano -sea cual fuere la forma en que se realice- es imposible sin el idioma.

* Estudiar psicológicamente al pensamiento como proceso, significa estudiar las causas internas que conducen a la formación de uno u otros resultados cognoscitivos.

El proceso del Pensamiento es ante todo análisis, síntesis y generalización.

* La Psicología, al estudiar el pensamiento al igual que cualquier otro proceso psíquico, considera en mayor o menor grado el estudio de las necesidades y los motivos que propician en la persona a que inicie esta actividad cognoscitiva, así como cuáles son las circunstancias concretas en que aparece la necesidad del análisis, síntesis etc.

Luria (5) precisa sobre la temática de el problema con respecto al pensamiento:

"Es sabido, que nunca percibimos los objetos del mundo exterior de manera aislada, sino asociados a determinados sistemas. Precisamente por esto, algunos investigadores (por ejemplo, Bruner 1957) dicen con total fundamento que percibir el mundo significa sistematizar los objetos que entran en él, codificar la información recibida.

Según la psicología clásica, los objetos percibidos del mundo exterior son asociados por el hombre en determinados sistemas lógicos en función de su cercanía, su semejanza o su ubicación en determinadas categorías generales.

Las teorías fundamentales acerca de la estructura de los procesos del pensamiento, empezando por los asociacionistas y los representantes de la escuela de Wurzburgo y terminando por los trabajos contemporáneos que se basan en la teoría de la información y la toma de decisión, se basaban en tales concepciones".

En resumen, de los procesos cognoscitivos (sensaciones, percepciones, memoria, pensamiento e imaginación) el pensamiento tiene prevalencia en razón a que:

- Es un proceso que está socialmente condicionado, lo que posibilita tener conciencia de lo que acontece en la realidad que lo rodea y con los contextos de su interés que desea relacionarse.

- Su naturaleza estratégica de referenciar el pasado y visualizar el futuro, estando en el presente.

- Crea mecanismos de autoevaluación en un marco normativo axiológico comportamental.

- Tiene su capacidad de procesar información y conocimiento en cantidad y calidad durante el trabajo de elaboración del plan estratégico.

Para el segundo nivel de trabajo que aborda la naturaleza estructural del planeamiento como proceso en una perspectiva psicológica de los procesos cognoscitivos, seguiremos tomando del contexto de la Administración como referente de entrada a través de la Teoría 
de las Organizaciones (TO) y la Teoría General de la Administración-TGA (1) con respecto a la dimensión del pensamiento.

Sobre la planeación como función administrativa, tenemos los planteamientos: Cit (c)

Según Urwick:

- "los elementos de la Administración son siete: investigación, previsión, planeación, organización, coordinación, dirección y control, que constituyen la base de una buena organización.

- Desdoblamiento para una mayor claridad del primer elemento de Fayol, la planeación, en tres fases distintas: investigación, previsión y planeación.

- La empresa no puede desarrollarse en torno a personas, sino a su organización”.

Según Gulick:

- $\quad$ "Los elementos de la Administración son siete (POSDCORB).

- La planeación es la actividad de trazar las líneas generales de lo que debe hacerse y fijar los métodos de hacerlo, con el fin de alcanzar los objetivos de la empresa".

Según el enfoque neoclásico (1935-1974):

- $\quad$ "la planeación es la primera función administrativa por ser la que sirve de base a las demás y permite a la organización evitar la improvisación.

- la planeación es la función administrativa, que determina por anticipado cuáles son los objetivos que deben alcanzarse y qué debe hacerse para conseguirlos".

A finales del siglo XX, el proceso administrativo, bajo una perspectiva global de Koontz, H. y Weihrich, H., determina cinco funciones administrativas: "la planeación", organización, integración de personal, dirección y el control de las actividades de los entes de la organización y empleo de todos los recursos con el objeto de alcanzar las metas establecidas; estas funciones de enfoque sistémico aparecen en el Cuadro $\mathrm{N} .^{\circ} 1$. 
Cuadro N. ${ }^{\circ}$ 1. Administración: Una perspectiva global.

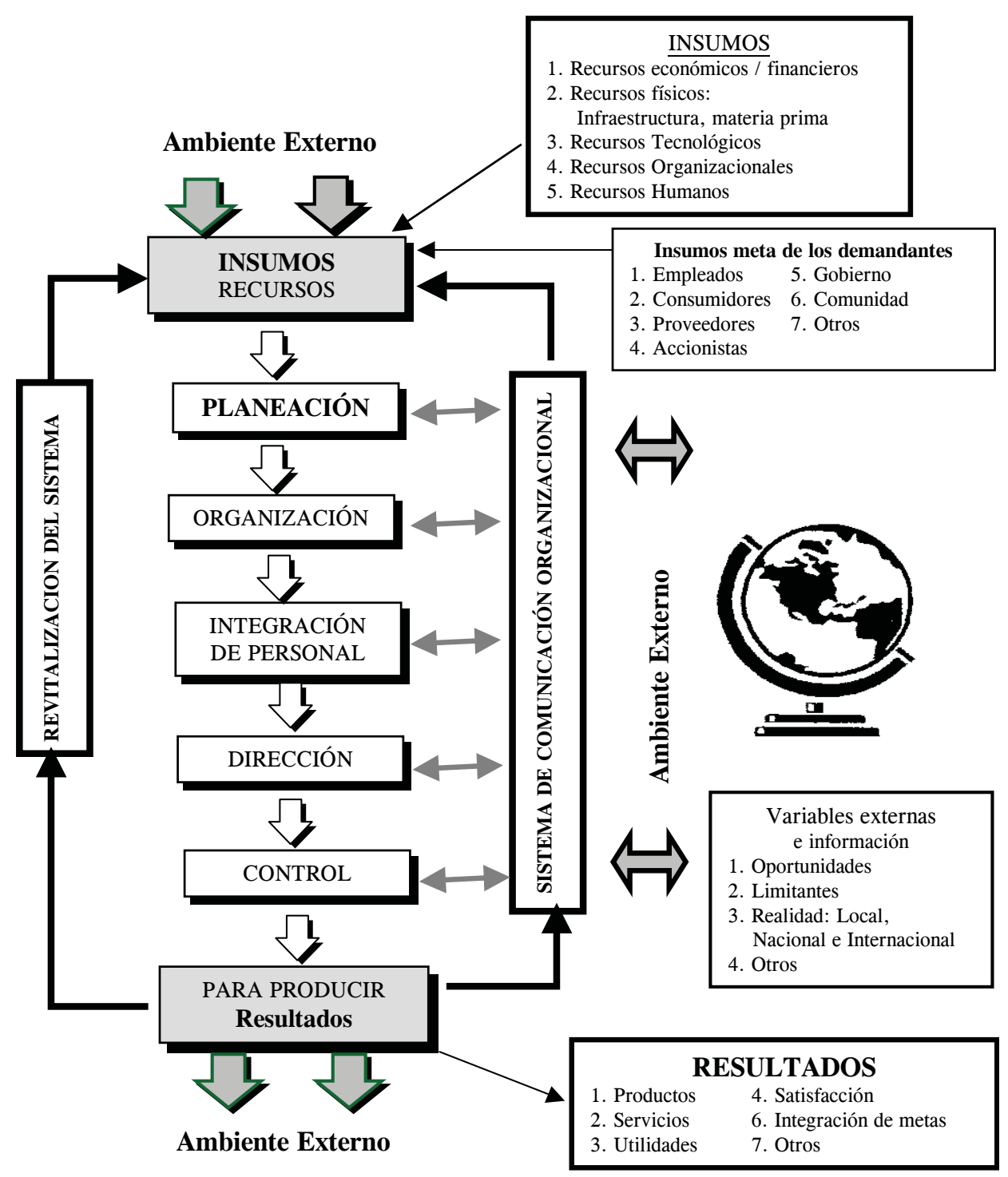

"Al diseñar un ambiente para el desempeño eficaz de las personas que trabajan en grupos, la tarea más importante de un administrador es asegurarse de que todas comprendan los propósitos y objetivos del grupo y sus métodos para lograrlos.

Para que el esfuerzo del grupo sea eficaz, las personas deben saber lo que se espera de ellas. Ésta es la función de la planeación, es la más básica de todas las funciones administrativas" (6). 
La planeación incluye seleccionar misiones y objetivos, y las acciones para alcanzarlos; requiere tomar decisiones: es decir, seleccionar entre diversos cursos de acción futuros.

La planeación permite salvar la brecha que nos separa del sitio a dónde queremos ir, hace posible que ocurran cosas que de lo contrario no hubieran sucedido. Aunque pocas veces se puede predecir el futuro con exactitud y aunque factores fuera de nuestro control pueden interferir con los planes mejor preparados, si no se planea, se deja que los sucesos ocurran por casualidad.

Así la planeación provee un enfoque racional para lograr objetivos preseleccionados, y también implica, intensamente, la innovación administrativa, y como proceso requiere de un esfuerzo intelectual; que determina conscientemente los cursos de acción a seguir y base para que las decisiones sean propósitos, conocimientos y estimaciones bien estudiados.

\section{MÉTODO}

La diversidad en lo conceptual y de los enfoques determina que la investigación sea exploratoria, que posibilite propuestas y modelo, e incremente las investigaciones.

\section{PROPUESTAS Y MODELO}

Esta realidad plantea la necesidad de evaluar y sistematizar enfoques para incrementar las investigaciones en base a propuestas sobre la efectividad de intervenciones que permitan la aplicación del planeamiento estratégico en la dinámica individual.

Esto determina que el Planeamiento Estratégico es una herramienta útil para el Psicólogo en el plano individual, que como profesional es su competencia la evaluación, intervención y prognosis de los procesos psicológicos, que en esta situación específica corresponde al proceso cognoscitivo del pensamiento, hacia sus clientes: personas y organizaciones de producción de bienes y servicios; extensiva particularmente por su utilidad al campo clínico, educativo, social comunal y político.

Para el propósito y fines que se persigue sobre la temática, es necesario resaltar referencias que a través de la evolución histórica de la aplicación del Planeamiento Estratégico, permita observar y evidenciar que desde la aparición de esta valiosa herramienta de gestión en las organizaciones, su elaboración principalmente, y en su administración y control, está directamente relacionado con los procesos cognoscitivos del ser humano, los que son de manejo principal y porque no expresarlo exclusivo, tanto por la formación científica y su capacidad técnica de los profesionales de la Psicología.

En esta búsqueda de referentes significativos a través de la evolución histórica del Planeamiento Estratégico en una perspectiva psicológica, nos situaremos en el siglo XX, con la aparición de lo tratado en el libro de Administración Estratégica bajo un enfoque integrado (7), cuyo modelo fue desarrollado originalmente en Harvard Business School durante la década del 60, que también se le conocería como el modelo de ajuste de formulación e implementación de una estrategia que ayude a las organizaciones en establecer una ventaja competitiva, denominación que se asocia al nombre de Kenneth Andrews (cit. a). 
Este primer referente expone sobre el Planeamiento Estratégico, que:

"El tema de la planeación sigue siendo un componente importante para la mayoría de las definiciones sobre estrategia en el área de la Administración.

Por ejemplo (cit. b), se definió estrategia como "la determinación de las metas y objetivos básicos a largo plazo en una empresa, junto con la adopción de cursos de acción y la distribución de recursos necesarios para lograr estos propósitos".

En la definición está implícita la idea de que estrategia involucra planeación racional. De manera similar tenemos la definición (cit. c): la estrategia como el modelo o plan que integra las principales metas, políticas y cadenas de acciones de una organización dentro de una totalidad coherente.

De igual manera (cit. d), se definió estrategia como un plan unificado, amplio e integrado, diseñado para asegurar que se logren los objetivos básicos de la empresa.

Las definiciones de estrategia basadas en la planeación, entre los que han generado críticas a los sistemas formales de planeación como ayuda para la toma de decisiones estratégicas, se encuentran Tomas Peters y Robert Waterman (cit. e), autores del best seller En busca de la excelencia, de forma similar Mintzberg (cit. f), en la revisión del concepto de estrategia, sugirió que las estrategias emergentes pueden ser tan exitosas como las estrategias intentadas, producto de la planeación formal".

Continuando la búsqueda en una perspectiva psicológica de referentes del planeamiento estratégico, nos situaremos en las décadas del 70 y 80 del siglo veinte, viendo por conveniente considerar las experiencias por la praxis profesional que se realizaba mediante la aplicación del Desarrollo Organizacional (D.O.), cuyos planteamientos se expusieron en un contexto anterior a la globalización.

"El planeamiento estratégico es una actitud gerencial que se puede expresar -la forma en que la dirección de una empresa emplea eficientemente sus recursos a mediano y largo plazo-". Otro detalle interesante del planeamiento estratégico es ¿quiénes lo pueden hacer? Yo creo que toda la organización lo puede y debe hacer, las diferencias están en el alcance con que cada una lo hace. (8)

En mi experiencia el planeamiento estratégico está en relación directa con la delegación de los directivos.

El planeamiento estratégico está en relación directa también con la capacidad gerencial. Una forma de potenciar el crecimiento de la dirección, es incluir dentro de ella a las nuevas generaciones por la fuerte creatividad que aportan para el planeamiento. Aquí el reto es cómo armonizar la guardia vieja con la nueva generación, y todo esto repercute en el planeamiento.

El planeamiento estratégico contiene alta dosis de innovación, de creación, de oportunidades y de medición de riesgos y cuando más grande es la organización, más tiempo hay que dedicar a esto. Si no se hace así, mejor es no crecer.

La planificación a mediano plazo implica la delegación operativa, esto es fundamental e imprescindible. A mi juicio se requiere un proceso mínimo de un año para obtenerlo. 
Los que lo hacen de manera intuitiva aciertan, los que planean lo obtienen. El lanzamiento de objetivos suele ser un retiro especial no menor de dos días, se aísla a reflexionar y planear, planear en meses, años, en nuevas posibilidades, en resolver incertidumbres".

Cabe anotar, que el haber incluido este referente es por el criterio que aplicaba este especialista en D.O. (Desarrollo Organizacional) acerca de la mentalidad estratégica, precisando que:

"Aquí nos encontramos a un problema de Psicología más que de Administración en sí. Después de veinte años de observación de cómo manejan las personas el progreso, creo que este problema más es de comportamiento humano que de técnica administrativa o de conocimiento administrativo puro.

El promedio normal de los ejecutivos es de una mente encasillada. La creencia que no pueden salir de sus comportamientos mentales establecidos y creer que así es la vida. Claro que en esto tiene influencia el conocimiento, pero allí no radica todo.

Esto es un valioso complemento cuando ellos se renuevan, pero de lo que se trata, es de mover la percepción y la voluntad, para autosacudirse de las ideas del pasado.

La mentalidad estratégica, esto es, la variación del presente para posesionarse mejor en el futuro requiere de tranquilidad mental y ésta no puede existir, si la mente no queda definitivamente libre de tareas de bajo nivel u operativas.

Un gerente sin esta mentalidad simplemente estará siempre preocupado del futuro, pero no estará haciendo nada por él."

Ahora nos situaremos a mediados de la década del 90, fecha en que aparece la corriente de la Administración: una perspectiva global, planteada por Koontz, H. y Weihrich, H. (6), expuesta anteriormente como referente de entrada del segundo nivel del marco teórico.

Con respecto a los tipos de planes, se clasifican según los cursos de acción futuros, puesto que un plan abarca cualquier curso de acción futura, los que a su vez también son planes, por tanto, la clasificación de los planes son variados.

Por otro lado, los pasos en la planeación son de aplicación general en cualquier tipo de planeación, y corresponden a:

1) detección de las oportunidades,

2) establecimiento de objetivos,

3) desarrollo de premisas,

4) determinación de cursos alternativos de acción,

5) evaluación de cursos alternativos de acción,

6) selección de un curso de acción,

7) formulación de planes derivados,

8) expresión numérica de los planes a través del presupuesto.

Sin embargo, en la práctica, se deberán estudiar la factibilidad de los posibles cursos de acción de cada paso o etapa. En conjunto podemos observar que los pasos de la planeación constituyen un proceso racional para la consecución de metas, el logro de objetivos. 
En este valioso referente se anota sobre los papeles (roles) administrativos que popularizó el profesor Mintzberg, como también se pronuncia sobre las críticas que hiciera Mintzberg a la Administración Estratégica (7 cit. f), con respecto al enfoque de la planeación que supone que la estrategia de una organización siempre es el producto de la planeación racional, sugiriendo que las estrategias emergentes pueden ser tan exitosas como las estrategias intentadas, producto de la planeación formal.

Complementando a todo lo tratado en las fuentes referenciales e intentando una compilación bibliográfica indicaremos en forma resumida los tipos de planes:

a) La Planeación Estratégica, en su consideración como el plan maestro de una organización que da forma a su destino.

b) La Planeación Táctica, como la planeación que transforma los planes estratégicos de una organización en metas específicas, por unidad organizacional.

c) La Planeación Operativa, a la planeación conformada por procedimientos y acciones específicas para aplicarse en los niveles inferiores de una organización.

Llegando al término del camino de búsqueda de referentes, considero relevante ir orientándonos hacia la aplicabilidad de esta herramienta de gestión, para lo cual es necesario considerar la propuesta sobre La Gerencia Integral (9) con su mensaje de "no le tema a la competencia, témale a la incompetencia" en la cual incluye nuestra temática de interés, el Planeamiento Estratégico.

"En los años 50, el paradigma dominante era la organización, es decir, se creía que cualquier problema de la empresa se podía resolver mediante un estudio de la organización o de un cambio del organigrama. Pero la experiencia demostró que había empresas que, a pesar de ser bien organizadas, fracasaban.

Así que las ciencias administrativas buscaron otro paradigma.

En los años 60 y 70, fue la estrategia. Entonces los mismos asesores de la organización de los años 50, cambiaron de nombre, pasaron a llamarse asesores en planificación estratégica. Su idea era que la empresa que tuviera una estrategia superior a la de sus competidores era la que tendría éxito; las demás fracasarían. Sin embargo, poco a poco, se hizo evidente que una estrategia no es sino un plan de acción sobre el papel y que para llevarla a cabo son necesarias las personas.

Esta evidencia llevó a buscar otro paradigma para los años 80: el de la "cultura organizacional”. Según este, para que la empresa tenga éxito, no sólo necesita una buena organización y una buena estrategia sino un equipo de gente de imaginar, formular $e$ implementar la estrategia.

La organización es un grupo de personas en interacción.

¿Cómo optimizar a la vez el proceso de producción y ventas y el proceso humano?

La respuesta queda en otro paradigma de los años 80: la calidad total.

Pero ya la calidad total va pasando de moda, y varios temas compiten por el título de paradigma dominante en los años 90: el "empoderamiento" (del inglés empowerment), la -reingeniería- ... 
Más allá de las modas administrativas y de las mutaciones semánticas que enriquecen el lenguaje de la administración, conviene buscar los verdaderos temas inmutables que rigen el comportamiento de las organizaciones:

- El "marketing", puesto que sin mercado, no hay producto, y sin producto (bien o servicio) para vender, la empresa no tiene razón de ser.

- La "Psicología Organizacional", porque una empresa, ante todo, es un grupo humano con cultura y comportamiento propios.

- Las "finanzas", puesto que la empresa es una entidad económica en pos de rentabilidad para los accionistas.

- La "planificación", para que la empresa elija su futuro en vez de sufrirlo.

- La "competitividad", que es el fin de la acción empresarial.

A la larga sólo sobreviven las empresas competitivas.

La realidad de los problemas de la organización es "global"

La Gerencia Integral, es el arte de relacionar todas las facetas del manejo de una organización en busca de una mayor competitividad:

- La estrategia: para saber a dónde vamos y cómo lograrlo.

- La organización: para llevar a cabo la estrategia eficientemente.

- La cultura: para dinamizar la organización y animar a su gente.

La Gerencia Integral atribuye como fin a la acción empresarial: la competitividad, es decir, el éxito relativo, con respecto al desempeño de los competidores.

La Planificación Estratégica es el proceso que sigue el gerente integral para dirigir y controlar el futuro de su empresa.

El concepto de estrategia y el de planificación están ligados indisolublemente, pues tanto el uno como el otro designan una secuencia de acciones ordenadas en el tiempo, de manera tal que sea posible alcanzar uno o varios objetivos.

La planificación estratégica subyace en el manejo del cambio, labor que corresponde al gerente integral, puesto que el cambio atañe a los tres aspectos de la Gerencia Integral: estrategia, estructura y cultura.

En un nivel de resumen de los referentes tratados sobre la temática y sumando lo desarrollado en la práctica profesional (10), nos permite plantear un modelo en una perspectiva psicológica, que, en base a las ventajas que ofrece el Planeamiento Estratégico, por un lado, determine sus componentes básicos estructurales, y sea flexible para su constante aplicación por nuevos métodos, técnicas e instrumentos, y del otro, su naturaleza intrínseca como producto de los procesos cognoscitivos de la persona, y su viabilidad de aplicación en la dinámica individual y organizacional.

De esta manera, se evitará que el plan estratégico no sufra constantes modificaciones en su estructura modular por la aparición de nuevas técnicas e instrumentos, que siempre serán necesarias para la ejecución de cada uno de sus componentes-módulos. 
Ante el escenario que viene presentándose ad portas final del segundo lustro del siglo XXI, por los efectos de la globalización producidos por el desarrollo de la ciencia y tecnología en general (11), el Planeamiento Estratégico, como un modelo de gestión de enfoque sistémico, puede convertirse en una alternativa que posibilite el aseguramiento del desarrollo sostenible de las organizaciones.

En este sentido, el Planeamiento Estratégico como modelo tendría dos componentes:

- $\quad$ un subsistema de Investigación con módulos de Estudios y Planeamiento, y

- $\quad$ el subsistema de Coordinación con sus módulos de Ejecución y Control.

- $\quad$ El módulo de Estudios a cargo del análisis interno y externo, y aplicación de técnicas de diagnóstico, que en términos de resultados, es el Análisis de la Situación de la Organización.

- El módulo Planeamiento a cargo de la elaboración de la visión, misión, valores, objetivos, estrategias, que en términos de resultados, es la formulación del Plan Estratégico.

- $\quad$ El módulo de Ejecución a cargo de la implementación del Plan y planes operativos, que en términos de resultados, es el desarrollo del Plan Estratégico.

- El módulo de Control a cargo de la verificación o medición, y la comparación de logros, que en términos de gestión, es el seguimiento y evaluación de resultados.

El Planeamiento Estratégico como modelo de gestión sistémica tiene líneas de coordinación entre los módulos que aparecen en el esquema:

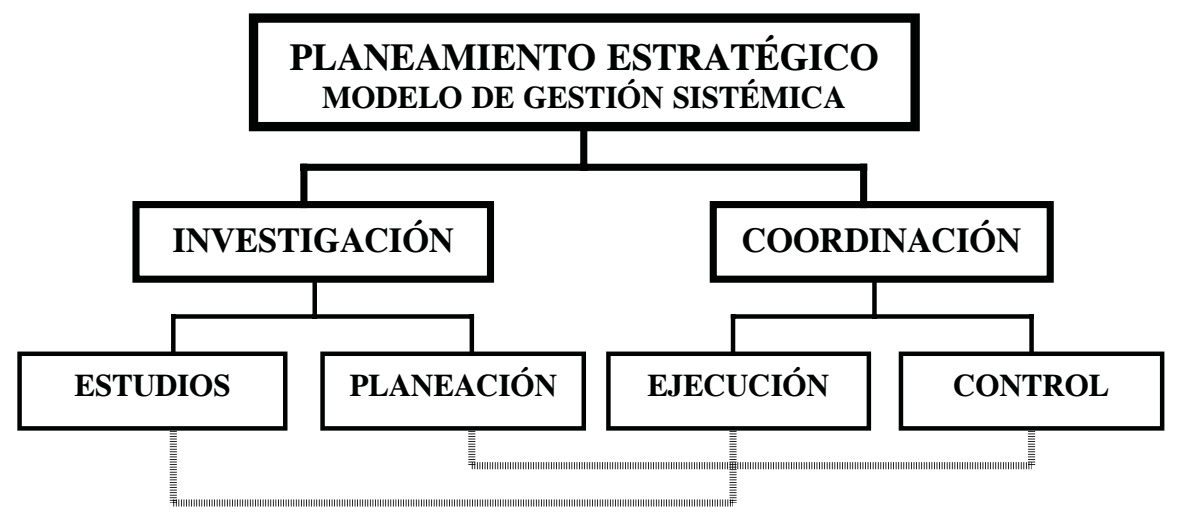

De esta manera, la Planeación será siempre un proceso que permita prever y decidir hoy las acciones que nos puedan llevar desde el presente hasta un futuro deseable, y que como proceso posibilita:

- Concretar ideas en planes y programas de actuación definidos en el tiempo y en el espacio.

- Asegurar el desarrollo presente y futuro de la organización.

- Identificar y solucionar estratégicamente problemas 
- Analizar la realidad interna y externa.

- Identificar y aprovechar oportunidades.

- Anticipar la atención de situaciones y tendencias del entorno.

- Consolidar y garantizar cumplimiento de la Misión.

- Formular objetivos verificables y medibles que se traducen en resultados.

- Conocer los pasos necesarios para lograr resultados previstos.

- Determinar o descubrir los obstáculos que posiblemente se opondrán al éxito y ver la forma de vencerlos.

- Contar con el elemento más valioso para el control.

- Tomar decisiones oportunamente.

- Contribuir al desarrollo del nuevo enfoque de gestión

- Mejorar competencias del personal.

- Favorecer la participación intra e intersectorial.

- Crear una red de alianza estratégica

- Contribuir al desarrollo de la organización.

La Planeación como proceso requiere para su desarrollo el ejercicio permanente del pensamiento estratégico de las personas, dando de esta manera capacidad para enfrentar los principales problemas y para mejorar el desempeño de la organización.

Asimismo, la Planeación fomenta y se apoya en la creatividad, iniciativa, innovación, y el liderazgo, revalorándolo para que transmita, convenza y haga participar a los demás en la gestión estratégica

La Planificación Estratégica es proactiva, orientada al logro de objetivos, basada en principios y valores y visión compartida.

La Planificación Estratégica se desarrolla a través de los siguientes niveles de trabajo:

- Nivel Enunciativo: al definir la misión y la visión

Para definir la visión parte de la consideración como declaración de valores, aspiraciones y metas, la fuente de autoridad y poder y el instrumento que imprime el liderazgo y la mística, conlleva un conjunto de valores.

Asumiendo que la visión es la imaginación mental de un futuro posible, deseable, realista, concreto, creíble, cargada de emoción y con fuerza de atracción.

Para definir la MIsión, parte de la consideración de reflejar el sentido de nuestro accionar, de declarar las principales capacidades y aspiraciones, de condicionar el análisis de las fortalezas, oportunidades, debilidades y amenazas, de generar un efecto cohesionador, de declarar la razón de ser de la organización, de ser el eje de la planificación estratégica y producto de la creación colectiva. 
- Nivel Explicativo: al realizar el análisis estratégico externo e interno, identificando, seleccionando y priorizando la atención de las situaciones-problemas. Se utilizará técnicas de diagnóstico organizacional, entre las cuales es de uso común la técnica del F.O.D.A.

- Nivel Normativo: Diseña los objetivos estratégicos.

Es el momento del "debe ser". Los Objetivos especifican los resultados que la organización desea alcanzar en un tiempo a través de la ejecución de la Misión.

En la construcción de los Objetivos se deberá responder a las siguientes interrogantes: ¿Qué cambiar?: situación a cambiar, ¿Qué se va a hacer?: acción

¿A quién? ¿Para quién?: población objetivo, ¿Dónde se desarrollará la intervención?: ámbito jurisdiccional, ¿Cuánto se va a hacer?: cuantificación del cambio, ¿Cuándo? ¿En qué plazo?: tiempo de aplicación

- Nivel Estratégico: Construye las estrategias y la viabilidad del plan.

La Estrategia como curso de acción son resultados de un proceso de análisis de contingencia situacional que conducen a la viabilización de un propósito, de un objetivo.

Nos indica la dirección a seguir para el logro de la misión. Es un modelo de decisión. Nos hace ser competitivos.

La Estrategia como curso de acción permite:

- cumplir con la Misión y Visión institucional;

- aprovechar las oportunidades;

- estructurar nuevas fortalezas;

- vencer las debilidades y anular las amenazas.

Para la viabilidad del Plan, se deberá responder a las siguientes interrogantes: ¿Quiénes serán los responsables?, ¿cómo lo haremos?, ¿qué recursos necesitamos?, ¿cuáles serán los recursos más adecuados?, ¿cuáles serán los plazos?

- Nivel Táctico Operacional: Programa la ejecución de actividades.

Determina la programación y las actividades táctico operativas.

- La Programación Táctico Operativa es un instrumento de gestión que, mediante la integración de información analítica, permite interrelacionar las actividades a ejecutar, con los responsables y los recursos a ser utilizados para el desarrollo de las mismas, en un periodo de tiempo predeterminado, organizada de tal manera que nos permita llevar a cabo nuestro objetivo de la manera más adecuada posible.

- Las Actividades Táctico Operativas son las actividades elegidas después de un proceso de análisis, en el entorno tiene enorme implicancia.

Se observan las variables determinantes o críticas que nos permitan elegir después del proceso, cuáles son las mejores alternativas para llevar a cabo nuestro objetivo. 
En los planes estratégicos institucionales se determinan también los Indicadores de Desempeño Estratégico.

Tratando de hacer una síntesis gruesa, arribamos que el Planeamiento Estratégico:

- es análisis y evaluación tanto de las oportunidades o limitaciones que ofrece el entorno de la empresa, como de la fortaleza y debilidades propias de la misma y se proyecta al futuro definiendo los objetivos, metas y estrategias que harán posible su consecución.

- es un proceso sistemático y permanente, que tiene un impacto significativo en el futuro de la empresa, significa decisión, riesgos empresariales y organización de los esfuerzos para ejecutar las decisiones, incluye equipos humanos multidisciplinarios y está sujeto a un proceso de evaluación permanente.

- es un proceso eminentemente participativo, que permite dirigir las actividades de la organización y los métodos de acción, prever los problemas antes que surjan y afrontarlos antes que agraven, contar con un sistema de información que ayude a conocer oportunidades y fortalezas.

- $\quad$ se expresa en un Plan Estratégico como producto resultante de la Planificación Estratégica que sirve de guía permanente de las acciones de cambio en la organización para el logro de resultados.

- que el Plan Estratégico en la perspectiva de cambio se le considera como la alternativa de atención de situaciones y/o solución de problemas.

- que el Plan Estratégico es una herramienta de gestión de ejecución de mediano y largo plazo.

En el campo de la aplicación de los profesionales de la salud la Planeación, Planificación o Planeamiento es un proceso de gran utilidad en la Atención del Cliente-Paciente-PAC, en el cual forma parte como una de sus etapas: Valoración, Diagnóstico, Planeamiento, Ejecución y Evaluación.

En la etapa de Planeación del Proceso de Atención del Cliente-PAC, se consideran las siguientes fases de trabajo: 1) Establecimiento de prioridades, 2) Elaboración de Objetivos. 3) Elaboración de Intervenciones y 4) Elaboración y compilación del Plan.

Al respecto, las organizaciones (empresas - industrias - instituciones) en su perspectiva para enfrentar el siglo XXI, dentro de un contexto globalizado y de ser parte de la dinámica de la sociedad del conocimiento, vienen realizando esfuerzos orientados a convertirse en organizaciones inteligentes (12), situación que les permitirá a unas ser exitosas, y a otras supervivir en el mercado tan competitivo, exigente y cambiante.

Es necesario tomar en cuenta el material sobre el Método de Casos como el cierre de las propuestas y modelo, para dar a conocer una de las técnicas muy interesantes que posibilitan entrenar al cerebro para pensar inteligentemente, de esta manera dar el primer paso si es que el empleado o ejecutivo aspira a ser miembro inteligente de una organización inteligente. 
La aplicación de la técnica del Método de Casos posibilita asumir una actitud permanente hacia el aprendizaje, a emerger la iniciativa y la creatividad en las personas, sean estudiantes o profesionales, trabajadores, supervisores, jefes, gerentes, ejecutivos o directivos, es decir, utilizar el método de casos como una herramienta para la toma de decisiones como práctica permanente se convertirá en una forma de comportamiento orientada a convertirse en gestores de organizaciones inteligentes.

Tomaremos como ejemplo la experiencia de una de las principales escuelas de negocios de nuestro país, del uso de la técnica del Método de Casos en la práctica académica con motivo de participar en el programa de desarrollo de los recursos humanos (13), y que se practicaba desde la década del 70 del siglo XX; posteriormente lo asumieron instituciones que desarrollan Programas de Alta Dirección y de Ejecutivos.

Este intercambio fecundo de pensamiento es uno de los aspectos más importantes del aprendizaje, basado en el método de casos y es sumamente efectiva para el aprendizaje, si se pone al estudiante en el papel del administrador que afronta el problema (o problemas, si el caso trata de varios).

El Método de Casos difiere de otros sistemas de enseñanza porque, a diferencia de los sistemas tradicionales, exige que la persona tome parte activa en el análisis de los problemas y en la determinación de alternativas o cursos de acción.

Los casos describen situaciones a las que se enfrentan las empresas y los hechos y opiniones de las que dependen los gerentes para tomar sus decisiones. El análisis de casos le permite aprender a través de la experiencia, y entre más casos resuelva, mayor será su habilidad para identificar problemas y formular soluciones, usted se beneficiará del análisis de casos en proporción a su esfuerzo.

Con el método de casos, las decisiones que usted haga y el proceso que usted siga para tomar decisiones es la clave, se espera que el sujeto desarrolle habilidades: para la toma de decisiones, para sustentar esas decisiones mediante un análisis adecuado, que aprenda a comunicar sus criterios, a defender los hechos y opiniones en debates, presentaciones o en informes escritos.

El desarrollo de habilidades analíticas y creativas para la solución de problemas, basadas en la experiencia obtenida al tratar con problemas tangibles, que han sido realmente afrontados por un administrador de negocios.

Su responsabilidad como analista incluye la participación, la interacción, la evaluación crítica y una comunicación eficaz.

El método de casos exige la participación individual en clase basada en una preparación cuidadosa del caso y no en un ejercicio de lectura rápida.

El método comprende las siguientes etapas:

1. Identificación del problema.

2. Recopilación de datos.

3. Lista de posibles soluciones. 
4. Prueba de las posibles soluciones.

5. Selección de la mejor solución.

6. Poner en práctica la solución.

Finalmente, el Psicólogo ejerce autoridad sobre la temática, al hacer uso de la información y el conocimiento, es decir, por el manejo profesional científico y tecnológico del proceso cognoscitivo del pensamiento, que es el insumo-recurso indispensable para desarrollar la aplicación del Planeamiento Estratégico, coadyuvando de esta manera al desarrollo personal de los seres humanos, de las organizaciones y del país.

\section{REFERENCIAS BIBLIOGRÁFICAS}

1. Chiavenato, Adalberto (1999). Introducción a la Teoría General de la Administración. Editorial Mc Graw Hill.

(cit. a) Bertalanffy, Ludwig von (1947). "The theory off Open Systems in Physics and Biology". Science, vol. 3.

(cit. b) Wiener, Norbert (1948). "Cybernetics or control and communication in the animal and the machine". The Technology Press of MIT. Cambrigde. Mass.

(cit. c) Urwick, Lyndall (1943). "The Elements of Administration". Paper on the Science of Administration.

2. Petrovski, A. Psicología general. Editorial Progreso.

3. Perea, J. L. (1998). "Los procesos cognoscitivos: Pensamiento". Separata curso anual Psicología y Psicopatología.- Facultad de Derecho de la Universidad Nacional Mayor de San Marcos.

4. Perea, J. L. (2002). "Psicología de la vida". Facultad de Educación, Universidad Alas Peruanas.

5. Luria, A.R. (1980). Los procesos cognitivos. Editorial Fontanella.

6. Koontz, H. y Weihrich, H. (1994). Administración: una perspectiva global. Editorial McGraw-Hill.

7. (cit. a) Hill, Ch. y Jones, G.- (1995). Administración estratégica: Un enfoque integrado. Editorial McGraw-Hill.

(cit. b) Chandler, A. (1962). Strategy and structure chapter in the history of the american enterprise.

(cit. c) Quinn, J. (1980). Strategies for change: Logical incrementalism.

(cit. d) Glueck, W. (1980). Business Policy and Strategic Management.

(cit. e) Peters, T. y Waterman R. (1982). In Search of Excellence.

(cit. f) Mintzberg, H. (1990). The design school: Reconsidering the basic premises of strategic management. 
8. Campoverde A. José. (1986). Gerencia eficaz mediante objetivos. Editorial Técnico Científica.

9. Sallenave, J.P. (2002). La gerencial integral. Editorial Norma.

10. Perea, José Luis. (2004). Procesos de gestión: Planeación”. Separata curso Psicología Organizacional. Facultad de Psicología de la Universidad Nacional Mayor de San Marcos.

11. Perea, José Luis (2007). “Administración y Globalización”. Revista del Instituto de Investigación en Psicología vol. 10 N. ${ }^{\circ} 1$. Lima: Facultad de Psicología, Universidad Nacional Mayor de San Marcos.

12. Senge, P. The fifth discipline: The art and practice of the learning organization". Editorial. Doubleday.

Senge, P. La quinta disciplina en la práctica: Estrategias y herramientas para construir la organización abierta al aprendizaje”. Editorial Granica.

13. ESAN. El Método de Casos. (AN176). 